\title{
e-Migrinter
}

$20 \mid 2020$

Hospitalité et migration

\section{Chibanis et chibanias ou l'(in)hospitalité au long cours}

Une comparaison France - Maroc

Julie Leblanc et Jordan Pinel

\section{OpenEdition}

Journals

Édition électronique

URL : https://journals.openedition.org/e-migrinter/2263

DOI : 10.4000/e-migrinter.2263

ISSN : 1961-9685

Éditeur

UMR 7301 - Migrinter

Référence électronique

Julie Leblanc et Jordan Pinel, «Chibanis et chibanias ou l'(in)hospitalité au long cours », e-Migrinter [En ligne], 20 | 2020, mis en ligne le 23 juillet 2020, consulté le 20 mai 2021. URL : http://

journals.openedition.org/e-migrinter/2263; DOI : https://doi.org/10.4000/e-migrinter.2263

Ce document a été généré automatiquement le 20 mai 2021.

Tous droits réservés 


\title{
Chibanis et chibanias ou l'(in)hospitalité au long cours
}

\author{
Une comparaison France - Maroc
}

Julie Leblanc et Jordan Pinel

1 Le contexte migratoire contemporain en Europe fait "crise » : "crise des migrants ", « crise humanitaire ", «crise de l'accueil », «crise de l'hospitalité ». Le terme de crise renvoie par définition à un phénomène brusque, aigu, nouveau. Ladite «crise migratoire » est ainsi médiatiquement et politiquement présentée en France. Elle n'est pourtant pas nouvelle. Nos travaux de recherche respectifs s'intéressent justement à des parcours migratoires anciens. Ceux de femmes et d'hommes aujourd'hui âgés de plus de 60 ans et qui ont émigré d'Algérie et du Maroc vers la France dans les années 1950-1960, seuls ou en famille. Ce qui est commun aux récits des personnes que nous avons pu rencontrer est la précarité et la vulnérabilité vécues lors de leur arrivée dans l'Hexagone et qui pour certains est toujours d'actualité. Toutes et tous ont connu les bidonvilles, les baraques, les garnis insalubres, l'hébergement précaire chez un proche, la faim, le froid, l'absence ou la rupture de droits, les contrôles de police et la peur. Ce statut précaire, lié à l'inhospitalité des cadres de l'accueil des immigrés en France, se traduit comme à son paroxysme par l'« encampement du monde» (Agier, 2011). Le "phénomène bidonville » est " presque consubstantiel du fait migratoire lui-même et (...) n'a par conséquent rien de nouveau " (Chavanon, Leblanc, 2018). C'est donc avec cette perspective historique que nous questionnons au sein de cet article le concept d'(in)hospitalité et ses implications dans la quotidienneté des personnes immigrées aujourd'hui âgées. À l'heure de la globalisation, nous sommes face à la dissémination et multiplication des situations de frontières - physiques, juridiques, sociales. Au-delà du caractère désuet que peut revêtir le terme d'hospitalité, nous l'entendons ici, à l'instar de F. Brugère et G. Le Blanc comme un "parcours" allant de "secourir " à « appartenir » en passant par « accueillir ». Ces auteurs rattachent les étapes et actions concrètes hospitalières comme nécessairement à chercher du côté de l'infra-politique et d'une "contre-politique hospitalière " (Brugère, Le Blanc, 2017). Face à des États 
inhospitaliers, les initiatives individuelles et collectives émergentes sont ici prises en compte.

Les résultats qui sont présentés dans cet article sont issus de nos travaux de thèse respectifs en anthropologie et géographie, proposant un double regard disciplinaire autour de la question du vieillissement des populations originaires du Maghreb (Algérie et Maroc). Nos enquêtes de terrain menées entre 2013 et 2019 en France (Lyon, Marseille) $^{1}$ et au Maroc (Agadir, Tiznit) ${ }^{2}$ permettent d'aborder différents ancrages sociaux et territoriaux. L'observation plus globale que nous obtenons, entre la France et le Maroc nous permet de mieux cerner les enjeux auxquels sont confrontés les populations migrantes vieillissantes, dans la société d'accueil et dans le pays d'origine. La réflexion qui s'est construite autour de ces questions a également permis de faire émerger des observations liées au genre, avec des terrains orientés exclusivement vers des femmes, ou à l'inverse essentiellement vers des hommes. Cette complémentarité permet de saisir au mieux les expériences de l'(in)hospitalité institutionnelle et les réponses apportées, notamment d'un point de vue associatif et citoyen. Ainsi, l'idée n'est pas pour nous de montrer les parcours de ces hommes et de ces femmes comme uniquement accablés par un contexte inhospitalier qui les écrase mais aussi de décrire l'agentivité à l'œuvre pour et par les personnes ici concernées.

\section{Pratiques d'(in)hospitalité : conditions de vie et droits sociaux des migrants âgés}

Pour les populations migrantes arrivées entre les années 1950 et 1970 en France, le vieillissement et le passage à la retraite est source de questionnements quant aux lieux où vieillir. Finalité du trajet migratoire, le retour finit souvent par constituer un "mythe » face à la prolongation du séjour dans la région d'accueil et l'enracinement progressif (Sayad, 1999). Les va-et-vient pratiqués par les migrants et notamment par les retraités compensent le non-retour et les rendent présents physiquement dans la société d'accueil tout en restant présents dans les processus qui ont cours dans leur société d'origine. Beaucoup de ces individus de «l'entre-deux» se sont forgés une identité multiple tout au long de leur migration (Schaeffer, 2001). Beaucoup deviennent des « transmigrants » qui « effectuent plusieurs séjours annuels au pays d'origine ou au moins un séjour annuel d'une durée supérieure à un mois " (Attias-Donfut, Gallou, 2006, p. 275-276). Mais ces maintiens en France constituent souvent « un renversement de la perspective classique de l'immigration, «disparaître » en tant qu'immigré en rentrant au pays pour pouvoir s'y investir ou bien « disparaître » en tant qu'émigré en s'incorporant à la société d'accueil » (Daum, 2007, p. 167). Montrant l'invisibilité des femmes face aux hommes dans ces mouvements migratoires associés aux travailleurs migrants des années 1960/1970, plusieurs travaux ont mis en avant le rôle essentiel des femmes dans les migrations originaires du Maghreb (Ait Ben Lmadani, 2012; El Hariri, 2003 ; Moujoud, 2010, Paiva, 2012). L'approche par le genre a apporté des éléments de compréhension de la vieillesse immigrée, soulignant le rôle des femmes dans la décision d'un non-retour définitif au pays d'origine, notamment dans leurs rapports aux enfants vivant en France :

«Pour la majorité des femmes, la possession d'une maison [au pays d'origine] ou la retraite du conjoint demeurent des raisons insuffisantes pour la réalisation d'un projet de retour définitif » (El Hariri, 2003, p. 47). 
Pour ces migrants arrivés le plus souvent comme source de main d'œuvre, la retraite comme synonyme de "vacance » n'allait pas de soi (Sayad, 2001). Travailler restait le seul moyen de ne pas subir l'assignation de "migrant inutile » par la société d'accueil (Arab, 2013). Cette retraite impensée et parfois impensable explique le manque de visibilité de cette population dans les politiques publiques. Ces dernières ne s'orientent d'ailleurs pas dans le sens d'une installation durable et d'une pratique de l'hospitalité. En ce sens, cette population envisagée comme "vouée au départ, dès son arrivée ", se trouve une fois retraitée face à des problèmes juridiques et sociaux, peu audibles notamment $\mathrm{du}$ fait du manque d'enjeu électoral qu'elle représente. Un associatif militant rencontré à Agadir explique ainsi que «les chibanis sont une population silencieuse car ils ne peuvent voter dans la société où ils vivent. Ils sont donc mis de côté.» (Agadir, Octobre 2017).

\section{De l'administratif au logement : I'inhospitalité au quotidien}

5 De nombreux travaux, ont analysé les processus sociaux d'enracinement des personnes immigrées en France en montrant en quoi ils influaient sur le non-retour au pays d'origine une fois l'âge de la retraite arrivé. Ainsi, le veuvage fréquent parmi les personnes interrogées (notamment chez les femmes), le nombre d'années de présence en France, les liens entretenus ou non avec le pays d'origine, leurs origines sociales et contextes de vie au moment de l'émigration, leur parcours socioprofessionnels en France, la présence ou non d'un entourage familial, leur situation économique et sociale ainsi que celle de leurs enfants, leur état de santé, le contexte local mais aussi national notamment $\mathrm{du}$ point de vue des politiques publiques ou de la législation, interfèrent de manière évidente au sein de leurs pratiques (Attias-Donfut, Gallou, 2006).

Au-delà, certains retraités et notamment les hommes seuls sont aussi astreints à l'immobilité par des procédures administratives qui empêchent leur retour ${ }^{3}$. Même si la pension de retraite peut être versée à l'étranger, diverses aides sociales comme l'Allocation de solidarité aux personnes âgées (Aspa) ne peuvent être touchées depuis l'étranger. Pour les bénéficiaires, il est donc nécessaire de rester au minimum 180 jours par an en France. De plus, les conditions de prise en charge des prestations liées à la santé sont, elles aussi, soumises à la condition de résidence en France. Ce contexte général n'incite pas au " retour » et participe à la précarisation de ces retraités lorsque ceux-ci choisissent malgré tout de vivre leur retraite dans leur pays d'origine. Une association marocaine ${ }^{4}$ située à Agadir vient ainsi en aide aux migrants âgés en situation de retour dans leurs démarches juridiques et administratives. Lors d'un entretien, voici ce qu'explique le président de l'association :

«Les gens qui ont travaillé en France pendant des années et des années, toute une génération, ils ont été travailler dans la mine par exemple, dans le charbonnage. Et ils y ont passé là-bas des années et des années et aujourd'hui ils ont des maladies, comme le retraité qui va arriver. Lui, il a une maladie du charbonnage, avec les poussières et tout ça dans les poumons après toutes les années qu'il a passé à respirer le charbon. Et des gens comme lui, il y en a beaucoup. Ils ont passé 30, 35 ans là-bas. Et après ils rentrent ici et ils se font soigner ici... sans assurance ! Donc c'est lui qui paie tous les médicaments. Il paie 130000 dirhams ${ }^{5}$ par an pour ses soins contre sa maladie du charbon. » (Agadir, Novembre 2017) 
7 L'histoire de ce retraité obligé de payer ses soins est symptomatique de la situation dans laquelle se retrouvent de nombreux travailleurs immigrés en France qui ont choisi de rentrer au pays. Pour bénéficier de la sécurité sociale française à laquelle ils cotisent, ils doivent résider en France au moins six mois par an. C'est donc le choix de beaucoup de retraités qui restent continuellement dans "l'entre deux", soit parce qu'ils souhaitent continuer à toucher des aides sociales, soit afin de pouvoir continuer à bénéficier de la sécurité sociale. Alors ils élaborent leurs calendriers de visite au pays en fonction de cette problématique des « six mois en France ». Ils le doivent car ils sont la cible prioritaire des contrôles pour suspicion de fraude en tant que ceux qui ont le plus intérêt à sortir du territoire français sur une longue période (Math, 2012). On le voit, qu'il y ait attachement ou pas au pays d'immigration, le retour au pays d'origine se révèle compliqué à mettre en œuvre face à cette absence de garantie de prise en charge de leurs besoins.

8 À Marseille, le quartier central de Belsunce est justement un espace qui peut être qualifié à la fois de transit (Temime, 1995) et d'installation pour ces chibanis qui font ces séjours pendulaires entre les deux rives de la Méditerranée. L'Algérie toute proche, se rejoint pour certains par bateau, " un simple sac plastique à la main pour le voyage ", confiait une responsable d'association du quartier. Les conditions de vie de ces travailleurs, aujourd'hui retraités, sont précaires voire indignes. Au cœur du quartier, dans une association dédiée à ces personnes âgées, une cinquantaine d'hommes de plus de 60 ans sont accueillis chaque matin pour un petit déjeuner gratuit. Il leur est proposé ensuite des permanences d'accès aux droits et à la santé. Ils vivent pour beaucoup dans le centre-ville de Marseille dans des hôtels, garnis et autres logements insalubres qui rappellent ceux de leur arrivée en France dans les années 1950. Leur santé est elle aussi précaire. Beaucoup ont du diabète, de l'hypertension sans compter les corps usés par le travail. Ils ont travaillé toute leur vie, parfois sans déclaration de la part de leurs employeurs, ce qui leur vaut une maigre retraite. Reconstituer leur carrière pour pouvoir accéder à leur dû est une des tâches ardues des travailleuses sociales de la structure que nous avons pu rencontrer. Pour une grande partie, cette pension est envoyée à leur famille restée en Algérie. Certains ont vécu et travaillé dans d'autres villes françaises puis ont choisi de vivre à Marseille une fois la retraite arrivée. Ceci non pas seulement pour le climat mais aussi pour les loyers et le coût de la vie plus faible qu'ailleurs pour se nourrir - dans le quartier Belsunce on peut accéder à un repas complet pour trois euros.

9 La comparaison entre le quartier Belsunce à Marseille et celui des Minguettes à Vénissieux (agglomération lyonnaise) fait ressortir des parcours de vie aux prises avec l'(in)hospitalité de manière différente. Dans les deux cas, les personnes sont passées par la précarité de l'habitat. Pour les personnes vivant aux Minguettes, l'accès au logement social bien que contraint par les politiques publiques, a été vécu comme bénéfique et le domicile comme le quartier ont pris après tant d'années valeur de « chez-soi ». Madame A. aujourd'hui âgée de 75 ans est arrivée d'Algérie en 1955, à l'âge de 12 ans. Alors que la guerre d'Algérie venait de commencer, sa famille l'a envoyée en France pour rejoindre ses frères et belles-sœurs qui étaient installés à Lyon. Elle s'est mariée en 1959 à 16 ans. Le couple s'est ensuite installé dans un garni au centre de Lyon. Puis ils ont vécu dans le bidonville du Chaâba nommé aussi « Bidonville du boulevard de ceinture » à Villeurbanne. Relogés après plusieurs années à la Cité Olivier de Serres construite dans les années 1950 pour les rapatriés de la guerre d'Algérie, Madame A. m'explique : 
Et là on a eu la belle vie là-bas. On avait un appartement. C'est la première fois qu'on voyait une salle de bain. L'eau chaude, les radiateurs et tout. Quand on est rentrés on s'est acheté notre première télé avec des boutons. J'ai installé mes enfants sur un matelas par terre, ils ont dormi comme des rois! On est restés pas mal de temps.

Ensuite, voyant que l'immeuble commençait à se dégrader, ils ont demandé à déménager :

On nous a proposé, La Duchère, Vaulx-en-Velin ou Vénissieux (Vénissieux, juin 2018).

Tous des grands ensembles périphériques construits à la fin des années 1960 .

Madame L., âgée de 80 ans et originaire de Sétif en Algérie évoquait avec ses filles le « luxe » que représentait l'accès aux logements de la ZUP des Minguettes : En premier on a vécu à Villeurbanne, on était les gones du Chaâba ${ }^{6}$, tu as lu le livre ? C'était nous! Puis ils sont arrivés aux Minguettes en 1968 : C'était l'Amérique!, me dirent-elles en évoquant la taille des appartements, et surtout le fait d'avoir plusieurs chambres, une salle d'eau et des toilettes (Vénissieux, juin 2013) (Leblanc, 2014, p. 82).

11 Toutes les femmes rencontrées vivent encore aux Minguettes parfois depuis la construction de la ZUP en 1969 et pour certaines dans le même appartement. Elles y ont élevé leurs enfants souvent nombreux dans des logements sociaux allant du T3 au T5. Aujourd'hui souvent veuves et vivant seules, elles subissent la pression des bailleurs qui souhaitent leur attribuer des logements plus petits. C'est le cas de Madame M. venue d'Oran à l'âge de 15 ans, aujourd'hui âgée de 81 ans. Elle est veuve depuis quatre ans et a six enfants qui pour la plupart vivent loin. Lors de notre première rencontre, elle m'explique que suite à une « réhabilitation » du quartier, leur tour avait été détruite et qu'elle et son mari avaient dû se battre pour avoir au moins un trois pièces pour pouvoir recevoir leurs enfants: $Y$ a des fois mes filles viennent, un F2 c'est, quoi ?! Une chambre, un salon... C'est quoi ?, me dit-elle alors en haussant les épaules (Vénissieux, juin 2013). Aujourd'hui, elle habite toujours seule dans son appartement de trois pièces. Malgré ses difficultés du fait d'escaliers à gravir pour accéder à son appartement, Madame M. n'ose pas demander à changer de logement de peur qu'on lui impose un deux pièces (Ibidem, p. 12).

Dans le centre de Marseille, la précarité des logements est toujours criante. Certaines personnes âgées suivies par l'association du quartier Belsunce sont aujourd'hui hébergées à l'hôtel suite à l'effondrement de leur immeuble. Les logements s'apparentent souvent à des chambres, parfois borgnes et sans ascenseur. Sur le cours Belsunce, les terrasses de café, ici y compris pour les femmes âgées, deviennent alors de véritables «extension de l'espace de l'habiter» (Ait Ben Lmadani, 2007, p. 232) pour sortir des quatre murs et de l'isolement.

\section{Risque d'isolement, les familles en tension}

Une dichotomie "Nord-Sud» quant aux représentations et au traitement de la vieillesse est fréquemment évoquée, dans laquelle les pays du Nord relèveraient d'une culture individualiste en opposition aux pays du sud considérés comme familialistes (Attias-Donfut, Gallou, 2006). Si « au regard des personnes âgées, aucune culture n'a le monopole de la tendresse et personne ne jette les siens » (Samaoli, 2007, p. 103), les données ethnographiques recueillies montrent la prégnance du modèle parfois 
injonctif de piété filiale en tant que norme (Attias-Donfut, Gallou, 2006, p. 154). Dans la pratique, les femmes plus souvent veuves du fait des écarts d'âge au moment du mariage et des carrières souvent usantes de leurs conjoints, vivent seules au domicile avec si besoin des aides infirmières et/ou ménagères. Si aux Minguettes, les enfants et les filles en particulier habitent souvent à proximité, à Marseille, beaucoup des hommes âgés rencontrés ont leur famille en Algérie. Les femmes quant à elles ont des parcours s'éloignant du schéma homogénéisant du regroupement familial. Certaines ont émigré seules, ont divorcé, n'ont pas d'enfant ou sont en ruptures familiales. Aussi, pour elles, les difficultés avec la langue française, notamment écrite, la complexité des démarches administratives à effectuer et aujourd'hui beaucoup dématérialisées, l'ignorance des droits et du tissu institutionnel et associatif, ainsi que les difficultés de mobilité, entraînent un risque d'isolement social important.

Un autre phénomène qui nuance une représentation familialiste attribuée à ces femmes, est ce que $C$. Roth nomme «l'inversion du contrat intergénérationnel » (Roth, 2010). Elle constate qu'au Burkina Faso, la paupérisation, l'inflation du chômage des jeunes, et les changements de modes de vie dont l'urbanisation, provoquent une aide des ainés envers les jeunes et non plus l'inverse. Ce constat se retrouve en France parmi les femmes rencontrées aujourd'hui âgées et souvent veuves qui subviennent toujours aux besoins de leurs enfants pourtant désormais adultes. Face à ce " cercle vicieux de l'appauvrissement " (Ibidem), la piété filiale est mise à mal et passe très souvent au second plan. Parmi les femmes rencontrées aux Minguettes, beaucoup hébergent encore leurs filles ou fils qui n'ont pas de travail ou de trop petits salaires. Elles gèrent très souvent l'intendance pour leurs enfants pourtant adultes. De même, plusieurs d'entre elles gardent leurs petits enfants pour aider leurs filles qui travaillent. Cette inversion explique en partie le phénomène de paupérisation des aînés et notamment des femmes qui parfois ne touchent qu'une pension de réversion. Ce phénomène est observable pour ces femmes comme d'ailleurs pour la population générale, et de part et d'autre de la Méditerranée.

\section{Ici et là-bas : des acteurs de l'hospitalité se mobilisent}

\section{L'émergence du tissu associatif en France}

15 Face à la situation qui peut être fragile en termes d'isolement social ou de conditions de vie des personnes immigrées âgées en France, les institutions peinent à se saisir de la question. Ces personnes, surtout lorsqu'elles n'ont pas de proches aidants, n'accèdent que peu aux droits sociaux. De plus, du point de vue de la sociabilité, elles ne fréquentent souvent pas les espaces dédiés aux séniors. Ceci pour plusieurs raisons : la faible résonance du concept de loisir ou en tout cas des loisirs proposés par ces espaces (bals musettes, bridge...) et l'accès onéreux à ces structures sont des facteurs repoussant pour les personnes rencontrées. D'autres espaces de sociabilité sont ainsi investis : les lieux de culte, les cafés (surtout pour les hommes), les associations dédiées aux familles comme les centres sociaux ou celles spécialisées dans l'accueil des personnes âgées immigrées telles les cafés sociaux ou structures d'accès aux droits.

Aux Minguettes, au sein des centres sociaux, l'une des activités développées à destination des adultes est celle des ateliers sociolinguistiques permettant aux personnes allophones une meilleure maîtrise de la langue française dans leur quotidien 
et leurs interactions sociales. La présence de femmes immigrées âgées de plus de 60 ans au sein de ces ateliers était importante au moment de leur mise en place dans les années 1990. Aujourd'hui, la succession des vagues migratoires mais surtout une réorientation des ateliers vers l'insertion par l'emploi ont écarté le public âgé devenu minoritaire. Pour autant, les femmes rencontrées comme les bénévoles et salariées des centres sociaux s'accordent sur les bénéfices en termes de sociabilité et d'apprentissage pour les plus âgées. Celles qui ont été mariées et dont le conjoint s'occupait de toutes les tâches extérieures au domicile (courses et démarches administratives notamment), sont confrontées au moment de la maladie et du décès de leur conjoint à des impasses au niveau du langage, surtout écrit. Quand je la rencontre, Madame M., est âgée de 81 ans. Elle est veuve depuis quatre ans et vit seule dans son appartement des Minguettes. Elle a six enfants qui pour la plupart vivent loin. Depuis un an elle suit des cours de français au centre social. Elle m'explique qu'elle n'est pas allée à l'école en Algérie mais qu'elle a appris à parler français en jouant avec les voisins de son âge. Arrivée en France à l'âge de quinze ans, elle me dit en souriant avoir régressé depuis qu'elle est ici. Même si elle parle très bien français, aujourd'hui étant veuve et vivant seule, son souhait précis est de pouvoir lire ce qui est écrit dans les magasins ainsi que les courriers, notamment les factures qu'elle reçoit. Elle m'explique qu'elle veut faire cette démarche depuis longtemps mais qu'elle n'a pas trouvé de lieu dédié aux personnes qui voulaient uniquement apprendre à lire et qui maitrisaient l'oral : «On me dit qu'il faut que mes petits-enfants m'aident mais...Je n'ai pas les petits-enfants avec moi» (Vénissieux, juin 2013). Aujourd'hui, notamment suite au terrain de recherche mené, un groupe de travail a été mis en place au sein de la structure pour réfléchir aux besoins spécifiques de cette population notamment en termes linguistique et d'isolement social.

Pour d'autres femmes rencontrées aux Minguettes, une association de culte nommée «mosquée » par les habitants du quartier, constitue un autre lieu d'hospitalité et de sociabilité. Au sein de cet espace les femmes âgées sont cette fois majoritaires. Elles s'y retrouvent pour certaines tous les après-midis. Elles y apprennent, à l'aide de femmes bénévoles, la lecture de l'arabe coranique mais aussi de l'arabe littéraire. Certaines mettent clairement en avant le lien social comme cette femme qui me disait «je veux sortir de ma bulle » ou encore Madame 0 . qui expliquait ainsi sa venue à la mosquée :

«On est allé au centre social, faire des activités tout ça, avec les enfants et tout. Et puis arrivée à la retraite, on tourne en rond, alors je suis venue ici » (Vénissieux, mai 2014) (Leblanc, 2014, p. 104).

Dans le cadre des sociétés d'immigration, où l'islam est minoritaire, « la mosquée remplit avant tout un rôle convivial qui révèle les liens parfois très forts qui peuvent se développer, en situation de migration ou de post-migration, entre personnes n'appartenant pas à la même famille mais fréquentant la même mosquée. Cette dimension met l'accent sur l'importance symbolique de la mosquée comme espace de convivialité et de solidarité » (Torrekens, 2009).

De plus, dans une logique associative et socio-éducative mais aussi de protection de la communauté musulmane en France, les mosquées s'ouvrent de plus en plus aux publics considérés comme fragiles tels les enfants et les femmes notamment âgées.

18 À Marseille, au sein du quartier Belsunce, une association a créé en 2008 un espace dédié à l'accueil des séniors du quartier. Ce projet a été créé par des militants et militantes sensibles à la précarité et aux discriminations dont les chibanis ont fait et font encore aujourd'hui l'objet en France. Le seul critère d'accès à la structure est d'être âgé d'au moins 60 ans. La forte proportion de personnes immigrées du Maghreb et 
d'Afrique sub-saharienne à Belsunce mais aussi l'accès à des professionnels parlant l'arabe et le wolof dans la structure, induisent une fréquentation majoritaire par les chibanis et chibanias. L'association a ainsi acquis au fil des années une expertise dans l'accompagnement spécifique de ces personnes, en termes de droits (assignation aux six mois par an en France, carrières difficiles à reconstituer), d'interprétariat mais aussi en termes de connaissance sociologique de cette population. Bien que très majoritairement fréquentée par des hommes, des femmes poussent la porte de l'association depuis plusieurs années. La plupart sont arrivées en France dans les années 1950/1970, mais d'autres sont arrivées tout récemment en France pour s'occuper de leur conjoint malade et qui ne peut rejoindre son pays d'origine pour les raisons déjà évoquées. À leur arrivée, la situation de ces femmes est alors très complexe : à la désillusion face aux conditions de vie précaires de leur conjoint avec qui parfois elles n'ont que peu vécu au quotidien, s'ajoute la non-connaissance totale du droit français, du tissu institutionnel et associatif mais aussi parfois de la langue. À cela s'ajoute les enfants et la famille qu'elles ont dû laisser dans le pays d'origine (Gallou, 2016). L'association devient alors pour elles à la fois un repaire et une ressource incontournable. Des moments et activités dédiés uniquement aux femmes sont ainsi mis en place par l'association, pour leur permettre d'accéder à un espace sécurisant où elles se sentent accueillies, écoutées et reconnues dans leurs difficultés.

\section{Un relais dans le pays d'origine}

L'exemple du Maroc nous montre que la région d'origine peut également devenir une source d'aide, un relais dans les problématiques sociales et institutionnelles que connaissent ces retraités. Un tissu social et associatif existe et se mobilise parfois, à leur niveau, pour apporter des réponses à l'isolement et aux ennuis administratifs.

Ainsi, nous avons pu rencontrer deux associations marocaines situées à Agadir qui, de deux manières différentes, tentent de répondre aux questionnements d'une population parfois égarée dans la "paperasse " administrative et dans leurs droits et non-droits. L'une est constituée de retraités ayant travaillé en Europe, en France principalement, mais aussi en Belgique, Allemagne ou Pays-Bas. Elle s'est constituée au début des années 2000 par des émigrés de retour s'apercevant que certains d'entre eux avaient des difficultés à faire valoir leurs droits face aux problèmes administratifs et aux incompréhensions. Ainsi, l'association s'est donnée pour mission d'orienter et de conseiller ces retraités de retour ou qui vivent encore en Europe, dans leurs démarches administratives, qu'elles concernent le pays d'accueil ou le Maroc. Plusieurs de ses membres de retour au Maroc ont décrit des situations «injustes » quant au fait qu'ils ne puissent être aussi bien couverts que s'ils étaient restés en France. Certains, encore résidents en France, laissent entendre que le retour n'était pas vraiment une option face aux conséquences sociales que cela pourrait engendrer : suppression de l'ASPA et soins médicaux non remboursés.

21 La seconde association rencontrée se revendique comme militante. Dirigée par un franco-marocain, fils de travailleur immigré en France, l'association milite pour les droits des travailleurs immigrés et notamment pour la possibilité d'un retour au pays avec conservation de l'intégralité des prestations sociales aujourd'hui non exportables. Peu présente sur le terrain, auprès des retraités, l'association milite essentiellement auprès d'hommes et de femmes politiques français ou marocains dans « un combat 
politique ». Celui-ci a contribué à un projet de loi qui a vu le jour en $2017^{7}$ en France, mais qui a échoué par manque de soutiens politiques. Loin de mâcher ses mots, le président de l'association qui connaît la situation de ces chibanis au quotidien, estime qu'on « laisse crever nos vieux migrants en France dans des conditions déplorables, sans se soucier de leurs droits » (Agadir, Octobre 2017).

Le soutien entre retraités migrants, se fait également en dehors des associations. Dans la région de Tiznit par exemple, beaucoup de retraités ayant travaillé en France se connaissent et se fréquentent au quotidien dans les cafés locaux. Ces cafés, lieux de sociabilité masculine, leur permettent de se retrouver entre personnes ayant eu des parcours migratoires similaires, ayant vécu dans les mêmes sociétés d'accueil. C'est dans l'un de ces cafés que nous avons pu rencontrer plusieurs d'entre eux. Ils sont de retour ou vivent encore en France et font un ou plusieurs allers-retours tous les ans. Ils expliquent que l'un de leur principal plaisir quand ils sont au Maroc, est de se retrouver entre amis de France, du Maroc et d'ailleurs pour prendre un thé ou un café, permettant de ne pas s'enfermer dans une forme d'isolement. Loin de constituer un groupe de parole ou de soutien de manière formelle, ces réunions permettent des échanges quotidiens, des conseils et des suivis des problèmes des uns et des autres. Ainsi, les discussions tournent souvent autour du «train-train » quotidien, mais aussi autour de questions plus techniques comme Mohamed qui ne comprend pas à qui envoyer le «certificat de vie » demandé par la caisse de retraite ou Abdelkbire qui veut savoir s'il peut demander un remboursement de ses médicaments achetés la veille.

Soutien moral comme soutien technique, les réseaux sociaux tout comme le réseau associatif viennent pallier les manques d'informations, les désinformations, les erreurs commises, mais surtout permettent de «faire communauté » pour une population qui se sent souvent mise à l'écart.

\section{En conclusion}

Les situations et initiatives abordées dans notre article confirment l'idée que face à un contexte global que nous considérons comme inhospitalier pour ces personnes immigrées retraitées, une "contre politique hospitalière " se met en place au niveau local par des acteurs concernés de près ou de loin par les questions migratoires, ceci de part et d'autre de la Méditerranée. Ces acteurs, lorsqu'ils sont associatifs, dépendent pour autant des politiques nationales et/ou européennes ne serait-ce que par le biais des subventions, ce qui les rend fragiles. L'incertitude des financements est renforcée par le fait que beaucoup des chibanis et chibanias n'ont pas la nationalité française et restent donc malgré une installation durable en France, dépourvus du droit de vote. Cela renforce leur invisibilité et leur inaudibilité sociales et politiques. D'autres acteurs, artistiques cette fois, se mobilisent autour de ces questions, par le biais du média filmique notamment comme le réalisateur Rachid Oujdi, décédé tout récemment, avec son film «Perdus entre deux rives, les Chibanis oubliés» (Oujdi, 2014) ou encore Nadir Dendoune avec son documentaire « Des figues en avril » rendant hommage à sa mère (Dendoune, 2018).

Les chibanis et chibanias eux-mêmes se mobilisent ici et là-bas pour faire valoir leurs droits. Pour exemple ce fut le cas collectivement en 2010 à Toulouse avec le soutien du collectif «Justice et dignité pour les chibanis ». Ces derniers ont occupé les locaux de la Carsat pour dénoncer ses contrôles discriminatoires (Host, Pépin, 2012). Plus 
récemment en 2018, la victoire des cheminots marocains ou le cas échéant de leurs veuves face à la SNCF, a permis de faire reconnaitre et réparer quarante ans de discrimination salariales. Bien que souvent décrits comme appartenant à l'âge de l'injonction à l'« invisibilité » et à la " politesse » (Bouamama in Guénif-Souilamas, 2006, p. 197), ces immigrés aujourd'hui âgés (et leurs enfants) ont de tout temps lutté pour leurs droits. Ce serait donc une erreur de les visibiliser uniquement sous l'angle de victimes. Comme le précise, Fatima Ait Ben Lmadani : l'«image de la personne âgée " sage ", respectée car faisant la prière et attendant en toute " sérénité » la mort, parait loin de la réalité » (Ait ben Lmadani, 2007, p. 337). En effet, les femmes immigrées marocaines qu'elle a rencontrées comme les femmes et les hommes côtoyés au cours de nos enquêtes respectives, continuent pour beaucoup à travailler, vivent seuls et produisent de multiples efforts pour acquérir une « reconnaissance sociale » que ce soit de la part des institutions françaises, de leurs pairs, de leurs sociétés d'émigration, ou encore de leurs familles.

Face à l'inhospitalité au long cours que nous avons observée et décrite dans cet article, des initiatives d'hospitalité et d'entraide sont mises en place. Mais elles le sont essentiellement par des collectifs associatifs et citoyens qui viennent pallier les défaillances de l'État. La reconnaissance des droits et des besoins des chibanis et chibanias fait son chemin, mais il reste encore beaucoup à faire et malheureusement le vieillissement, lui, suit son cours aussi.

\section{BIBLIOGRAPHIE}

Agier, Michel (2011) L'encampement du monde, Plein droit, vol. 90, n 3, pp. 21-24.

Ait Ben Lmadani, Fatima (2007) La vieillesse illégitime? Migrantes marocaines en quête de reconnaissance sociale, Paris, Université Paris-Diderot, $371 \mathrm{p}$.

Th. Doct. : Sociologie : Université Paris Diderot, URMIS : 2007.

Ait Ben Lmadani, Fatima (2012) Femmes et émigration marocaine. Entre invisibilisation et survisibilisation : pour une approche postcoloniale, Hommes \& Migrations, $\mathrm{n}^{\circ} 1300$, pp. 96-103.

Attias-Donfut, Claudine ; Gallou, Rémi (2006) L'enracinement. Enquête sur le vieillissement des immigrés en France, Paris, Armand Colin, 292 p.

Arab, Chadia (2013) Le troisième âge : le « migrant inutile », Hommes \& Migrations, n 1303, pp. 47-55.

Brugère, Fabienne ; Le Blanc, Guillaume (2017) La fin de l'hospitalité. Lampedusa, Lesbos, Calais... Jusqu'où irons-nous? Paris, Flammarion, $240 \mathrm{p}$.

Chavanon, Olivier ; Leblanc, Guillaume (2018) Immigration et habitat de fortune, une sempiternelle redécouverte. Écarts d'identité, n 130, pp. 8-11.

Daum, Christophe (2007) Migration, retour, non-retour et changement social dans le pays d'origine, In Petit, V. (Ed.) Migrations internationales de retour et pays d'origine. Paris, CEPED, pp. 157-169 (Rencontres). 
Dendoune, Nadir (2018) Des figues en avril. Film documentaire, 58 minutes.

El Hariri, Saâdia (2003) Les femmes et le retour au pays d'origine, Hommes \& Migrations, $\mathrm{n}^{\circ} 1242$, pp. 43-52.

Gallou, Rémi (2016) Vieillir sans conjoint mais vieillir entourées : un défi pour les femmes immigrées, Gérontologie et société, vol. 38, n 149, pp. 105-123.

Guénif-Souilamas, Nacira, (dir.) (2006) La république mise à nu par son immigration, Paris, La Fabrique, $151 \mathrm{p}$.

Host, Jérôme ; Pépin, Juliette (2012) Justice et dignité pour les chibanis, Plein droit, nº 93, pp. 8-11.

Math, Antoine (2012) Le contrôle par la résidence, Plein Droit, n 93, pp. 3-7.

Leblanc, Julie (2014) « Une diversité [in]visible? » Des femmes immigrées âgées dans la Cité, Lyon, Université Lumière - Lyon 2.

Mém. Master II : Anthropologie : Université Lumière Lyon 2 : 2014.

Moujoud, Nasima (2010) Genre et migration. Entre androcentrisme et prisme de 'la culture d'origine', Naqd, Revue d'études et de critique sociale, n² 28, pp. 55-75.

Oujdi, Rachid (2014) Perdus entre deux rives, les Chibanis oubliés. Film documentaire, $52 \mathrm{mn}$.

Paiva, Michelle (2012) Des femmes invisibles, Plein Droit, n 93, pp. 21-24.

Petit, Véronique (Ed.) (2007) Migrations internationales de retour et pays d'origine. Paris, CEPED, 208 p. (Rencontres).

Roth, C (2010) Les relations intergénérationnelles sous pression au Burkina Faso, Autrepart, $\mathrm{n}^{\circ} 53$ pp. 95-110.

Samaoli, Omar (2007) Retraite et vieillesse des immigrés en France. Paris, L'Harmattan, 276 p.

Sayad, Abdelmalek (1999) La double absence. Des illusions de l'émigré aux souffrances de l'immigré.

Paris, Le Seuil, 437 p.

Sayad, Abdelmalek (2001) La vacance comme pathologie de la condition d'immigré. Le cas de la retraite et de la pré-retraite. Revue Européenne des Migrations Internationales, vol. 17, n 1, pp. 11-36.

Schaeffer, Fanny (2001) Mythe du retour et réalité de l'entre-deux. La retraite en France, ou au Maroc ?, Revue Européenne des Migrations Internationales, vol. 17, nº 1, pp. 165-176.

Temime, Emile (1995) Marseille transit : Les passagers de Belsunce. Paris, Autrement, 139 p. (Série Monde - Français d'ailleurs, peuple d'ici),

Torrekens, Corinne (2009) L'espace de la mosquée à Bruxelles : nouveaux liens sociaux et investissement politique, Revue des mondes musulmans et de la Méditerranée, $n^{\circ} 125$, pp. 23-45.

\section{NOTES}

1. L'enquête ethnographique de Julie Leblanc débute en 2013 et s'appuie sur la rencontre de 48 femmes âgées de 59 à 83 ans, originaires majoritairement d'Algérie mais aussi de Tunisie, du Maroc et d'Afrique de l'Ouest. Les données ont été recueillies dans la zone urbaine des Minguettes à Vénissieux (Rhône) par le biais d'observations participantes dans une association d'habitantes et dans les centres sociaux du quartier en tant que bénévole dans des cours de français, mais aussi en tant qu'apprenante de l'arabe dans une mosquée des Minguettes. Des entretiens de récits de vie ont été recueillis auprès de ces femmes dans ces structures mais aussi 
au domicile de plusieurs d'entre elles. De plus, en 2018, dix ateliers de recherche partagée avec une artiste et une photographe ont été menés avec 14 de ces femmes et ont donné lieu à une exposition et un film retraçant la mémoire et le vécu de ses femmes au sein du quartier. À Marseille, en 2019, le terrain a été mené avec l'aide d'une association d'accès aux droits pour les personnes retraitées du quartier Belsunce. À nouveau, dix ateliers de recherche partagée ont été menés ainsi que des entretiens de récits de vie avec 17 femmes et en partenariat avec une documentariste sonore pour retracer le vécu de ces femmes et leur vision du quartier. Une journée de rencontre entre les femmes des Minguettes et de Belsunce a été organisée en juin 2019.

2. L'enquête de terrain de Jordan Pinel s'est déroulée entre 2017 et 2019 dans la région SoussMassa (Maroc) et s'est centrée autour d'une approche biographique avec des matrices reprenant les aspects résidentiels et familiaux des mobilités, qui constituaient le cœur de l'enquête. Un entretien semi-directif suivait la grille afin d'apporter des nuances et précisions permettant de mieux comprendre les interactions et interférences entre les évènements biographiques des individus et ceux de leurs proches. L'échantillon se compose de 20 Marocains et FrancoMarocains (tous nés Marocains au Maroc et ayant acquis la nationalité française) : 18 hommes et 2 femmes. En outre, une dizaine d'entretiens supplémentaires ont été effectués auprès d'acteurs institutionnels, associatifs et privés "gravitant» autour de la présence de ces retraités: des représentants de fondations ou ministères marocains centrés sur la question des Marocains Résidant à l'Étranger, deux associations de Marocains émigrés à l'étranger ou retraités, la CNSS (Caisse Nationale de Sécurité Sociale) de Tiznit.

3. Le numéro 93 de la revue Plein Droit, «Vieillesse immigrée, vieillesse harcelée » fait état de ces restrictions.

4. Dans un souci d'anonymisation, les noms des associations citées dans cet article ne seront pas mentionnés.

5. 130000 dirhams sont équivalents à environ 12000 euros.

6. En référence à l'ouvrage d'Azouz Begag publié en 1986, sous le titre Les gones du Chaâba, racontant son enfance dans ce bidonville de Villeurbanne.

7. Projet de loi ${ }^{\circ} 4412$ proposé à l'Assemblée Nationale le 25 janvier 2017 et « visant à améliorer les conditions de vie et de séjour des Chibanis et de leurs familles ».

8. Voir à ce sujet l'article du Monde, daté du 17 avril 2018, « Les chibanis discriminés l'emportent définitivement face à la Sncf». Source: https://www.lemonde.fr/police-justice/article/ 2018/04/27/les-chibanis-discrimines-l-emportent-definitivement-face-a-la-

sncf_5291683_1653578.html (consulté le 17 mai 2019).

\section{RÉSUMÉS}

Nous questionnons au sein de cet article le concept d'(in)hospitalité et ses implications dans la quotidienneté des personnes immigrées aujourd'hui âgées en France. Les enquêtes qualitatives qui sont mobilisées conduisent à s'intéresser particulièrement au vieillissement des chibanis et des chibanias, ces immigrés venus en France souvent dans le cadre de dispositifs migratoires étatiques à partir des années 1960. Certains font face à des conditions de vie précaires et des droits sociaux très limités, illustrant les pratiques inhospitalières des institutions. Face à ce constat, nous observons que ce sont alors les acteurs locaux de l'hospitalité, souvent associatifs, qui se mobilisent pour contrebalancer des situations perçues comme injustes. Les personnes 
concernées s'impliquent également elles-mêmes pour (re)créer des espaces d'hospitalité et d'entraide dans des lieux tels que les cafés mais aussi pour conquérir et faire valoir leurs droits et la reconnaissance sociale qui leur fait défaut. Ces acteurs et actrices de la société civile sont mobilisés « ici » et « là-bas », c'est-à-dire autant dans le pays d'accueil que celui d'origine - le Maroc dans ce cas d'étude - rappelant la vie «d'entre-deux » pour une partie de ces populations aujourd'hui vieillissantes.

\section{INDEX}

Index géographique : France, Algérie, Maroc

Mots-clés : associations, personnes âgées, accès aux soins, accueil, vie quotidienne, Chibanis

\section{AUTEURS}

\section{JULIE LEBLANC}

Doctorante en anthropologie, LADEC (Fre 2002) Université Lumière Lyon 2

Fellow IC Migrations (2018 - 2021)

julie.leblanc@univ-lyon2.fr

JORDAN PINEL

Doctorant en géographie, Laboratoire MIGRINTER (UMR 7301) CNRS / Université de Poitiers

jordan.pinel@univ-poitiers.fr 